\title{
The Interaction Between the Patient and Nurse Anesthetist Immediately Before Elective Coronary Artery Bypass Surgery
}

\author{
Katarina Berg, Rikard Kaspersen, Christina Unby and Gunilla Hollman Frisman
}

\section{Linköping University Post Print}

\section{Tweet}

N.B.: When citing this work, cite the original article.

Original Publication:

Katarina Berg, Rikard Kaspersen, Christina Unby and Gunilla Hollman Frisman, The Interaction Between the Patient and Nurse Anesthetist Immediately Before Elective Coronary Artery Bypass Surgery, 2013, Journal of Perianesthesia Nursing, (28), 5, 283-290.

http://dx.doi.org/10.1016/j.jopan.2012.10.008

Copyright: WB Saunders http://www.elsevier.com/

Postprint available at: Linköping University Electronic Press http://urn.kb.se/resolve?urn=urn:nbn:se:liu:diva-100911 
The interaction between the patient and nurse anesthetist immediately prior to elective coronary artery bypass surgery

Berg Katarina ${ }^{1}$ CRNA, Lic.Med., Kaspersen Rikard ${ }^{2}$ CRNA, MScN., Unby Christina ${ }^{3}$ CRNA, MScN., Hollman Frisman Gunilla ${ }^{1,4}$ CCRN, PhD, Ass Prof.

${ }^{1}$ Division of Nursing Sciences, Department of Medicine and Health, Faculty of Health Sciences, Linköping University, Linköping, Sweden. ${ }^{2}$ Sykehuset Østfold HF, Anestesiseksjonen, Avdeling for anestesiologi og operasjon, 1903 Fredrikstad, Norway.

${ }^{3}$ Sirius Humanum AB, Gamla Övägen 25, 60379 Norrköping, Sweden. ${ }^{4}$ Anaesthetics, Operations and Specialty Surgery Centre, County Council of Östergötland, 58185 Linköping, Sweden.

Corresponding author: Gunilla Hollman Frisman CCRN, Ass. Prof., Division of Nursing Sciences, Department of Medicine and Health, Faculty of Health Sciences, Linköping University, Linköping, Sweden. E-mail: Gunilla.Hollman.Frisman@liu.se, Phone: +46 10103 2115. 


\begin{abstract}
Establishing a purposive interaction with a patient who is in a vulnerable situation prior to coronary artery bypass graft $(\mathrm{CABG})$ surgery poses a challenge for the nurse anesthetist. The aim of this study was to identify and describe the interaction between the patient and nurse anesthetist immediately prior to elective CABG surgery. An observational study including 11 situations where the patient and the nurse anesthetist interacted was performed. A theoretical selection of patients and nurse anesthetists was made and data were analyzed using constant comparative method. The core category, Reassurance, emerged from the categories Continuity, Confirmation and Control. Continuity was characterized by expedient anesthesia nursing of high quality, Confirmation was related to communication in a trusting atmosphere and Control was associated with skilled nursing interventions in the thoracic operating theatre. In conclusion, reassurance can be achieved through a well-structured anesthesia nursing performance at the thoracic operating theatre, and by focusing on the patient and continuously giving him/her information during the preoperative preparation.
\end{abstract}

Keywords: coronary artery bypass graft surgery, grounded theory, interaction, nurse anesthetist 


\section{INTRODUCTION}

The idea behind nursing care is to alleviate the patient's suffering by planning the care according to the patient's resources and needs [1] and to facilitate a purposive patient-nurse interaction [2]. In an anesthesia context, nursing care relies on the interaction between the patient and the nurse anesthetist during which the nurse makes his/her competence visible to the patient when they connect with each other [3]. The starting point of the interaction is the establishment of a relation in which the patient and the nurse get to know each other $[4,5]$. The interaction is built on respect and empathy [3], concepts essential to a professional nursepatient relation in an anesthesia context as well. Respect in an acute and technical setting is based on the nurse being prepared, able to make the patient comfortable and clearly conveying that he/she is there for the patient [6]. In an anesthesia environment, empathy is facilitated by understanding who the patient is $[7,8]$ and identifying any concerns the patient may have [7]. The interaction between people can be viewed from the perspective of symbolic interaction, where the concept of meaning is one major element in understanding human behavior [9]. Symbolic interaction is based on three factors: Firstly, humans act on the basis of meaning. Secondly, meaning is derived from, or arises from, the social interaction between human beings. Finally, meaning is modified through an interactive process used in every interaction between human beings $[9,10]$. In a highly qualitative interaction between a patient and a nurse in an acute care setting, meaning is based on the nurse being flexible in changing between physical- and psychosocial care [11].

Communication and attitudes are essential in the interaction between the nurse anesthetist and the patient [12]. Mead [10] expresses that we see ourselves indirectly through the response of others and act with reflections of one another. We see through the other person's eyes and contemplate our own reactions. This interaction establishes a relationship between the patient and the nurse, where the nurse is responsible for the interaction as the patient is dependent on 
the nurse's competence and experience [13]. To establish a confident relationship before anesthesia induction, the nurse anesthetist constantly interacts with the patient in order to develop intimacy and reduce the psychological stress response [8].

For many patients afflicted by ischemic heart disease and waiting for coronary artery bypass graft surgery $(\mathrm{CABG})$, the preoperative situation is distinguished by existential concerns of suddenly losing their life in a heart attack [14]. Experience of physical pain combined with thoughts of life and death may induce a feeling of insecurity. The patient tends to worry and experience a sense of loneliness and lack of freedom, resulting in a decreased quality of life $[14,15]$. In anesthesia nursing care, patient centered care is mediated through safeguarding the patient and being responsible for the patient throughout the surgical procedure [7], thus supporting the patient's wellbeing [3].

In the immediate preoperative situation, the patient's explicit and implicit expectations, concerns and needs are met by the nurse anesthetist's professional competence during a restricted time of interaction. Due to limited nursing research of the interaction between the patient and nurse anesthetist during this restricted time course, this study aims to expand the understanding of their interaction.

\section{Purpose}

The purpose of this study was to identify and describe the interaction between the patient and nurse anesthetist during the immediate preoperative preparation prior to elective $\mathrm{CABG}$ surgery. 


\section{METHODS}

To understand the interaction between the patient and the nurse anesthetist prior to elective CABG surgery, an observational study using constant comparative method was performed. As the interaction between the nurse and the patient is regarded as a social process, grounded theory which is rooted in symbolic interaction and aims to understand how people interact with each other was found to be an appropriate method [16]. Observations provide an opportunity to understand the symbolic world of the interaction between the patient and nurse anesthetist [17, 18]. The present study was approved by a Regional Ethical Review Board and was carried out in accordance with the Declaration of Helsinki.

\section{Setting}

The study was carried out at a cardiothoracic operating theatre at a University Hospital in Sweden. A total of 17 nurse anesthetists worked at this theatre, all but two female. The median experience as a nurse anesthetist was 22 years. They worked in close teams with theatre nurses, assistant nurses, anesthesiologists and surgeons. The patients were rigorously informed about their disease and the surgical procedure before surgery. Information was given verbally and through brochures by the ward nurse, the anesthesiologist and the surgeon. All patients had also been offered to watch an information video about expected peroperative procedures. The video showed the operating theatre environment, how the staff was dressed and all procedures prior to the surgery. It also contained information about the postoperative period in the recovery room. When the patients arrived in the operation theatre they had received premedication according to local guidelines, mostly acetaminophen and benzodiazepines. 


\section{Sample}

To find similarities and differences and widen the discovery of categories, an appropriate theoretical selection was made. Patients were theoretically selected from a list of patients scheduled for elective CABG surgery, based on maximum variation in age and gender. The nurses were likewise selected but on the basis of their working schedule and gender. The theoretical selection continued throughout the data collection until saturation was reached [16].

All patients scheduled for elective CABG surgery were informed about the study through a patient information letter and by the nurse at the ward on arrival the day before surgery. The patient information letter included the purpose of the study, information that observations may be performed on the day of surgery and that the care would not be affected by the ongoing study. The confidential and voluntary nature of participation was stressed. The letter also included an informed consent. The nurses were verbally informed at a staff meeting that observations might be performed. They also received a study information letter which contained information on the confidential and voluntary nature of participation along with an informed consent. Before each observation the authors ensured that both the patient and the nurse anesthetist had signed the informed consent.

\section{Data collection}

In order to familiarize themselves with the surroundings and the nurse anesthetists, two of the authors (RK, CU) were present at the cardiothoracic operating theatre for a few days between November 2009 and January 2010. No observations were made during this period. Data were collected through unstructured observations from February to April 2010. During the 
observations the authors acted as complete observers standing at a range of 1 to 1.5 meters from the patient so that the interaction could be observed the whole time.

Two pilot observations were performed to evaluate the method, out of which one was included in the findings. The other pilot observation was excluded due to the observer becoming involved in the situation and interacting with both the nurse anesthetist and the patient. When the first observation had been analyzed by all authors, the next situation was theoretically selected in accordance with grounded theory. Thereafter, one observation at a time was performed and a theoretical selection was made following analysis and comparison with the previously analyzed data [17].

To minimize bias, observations were made by one observer at a time. The observations took place in hallways and operating rooms. They started when the patient arrived in the operating theatre and went on until he/she was asleep before the surgery. The observations lasted for 20 to 40 minutes. To avoid missing essential pieces of the interaction, no field notes were made during the observation $[17,18]$. Instead, extensive field notes were written down immediately after each observation. No further observation was made before the field notes had been analyzed and compared with the previously analyzed data. A total of 11 patients, 6 men and 5 women and 10 nurse anesthetists, 2 men and 8 women, were observed in 11 situations. One nurse anesthetist was observed on two occasions by different observers; observation numbers 2 and 8 (Table 1). 
Table 1. Characteristics of observed patients and nurse anesthetists

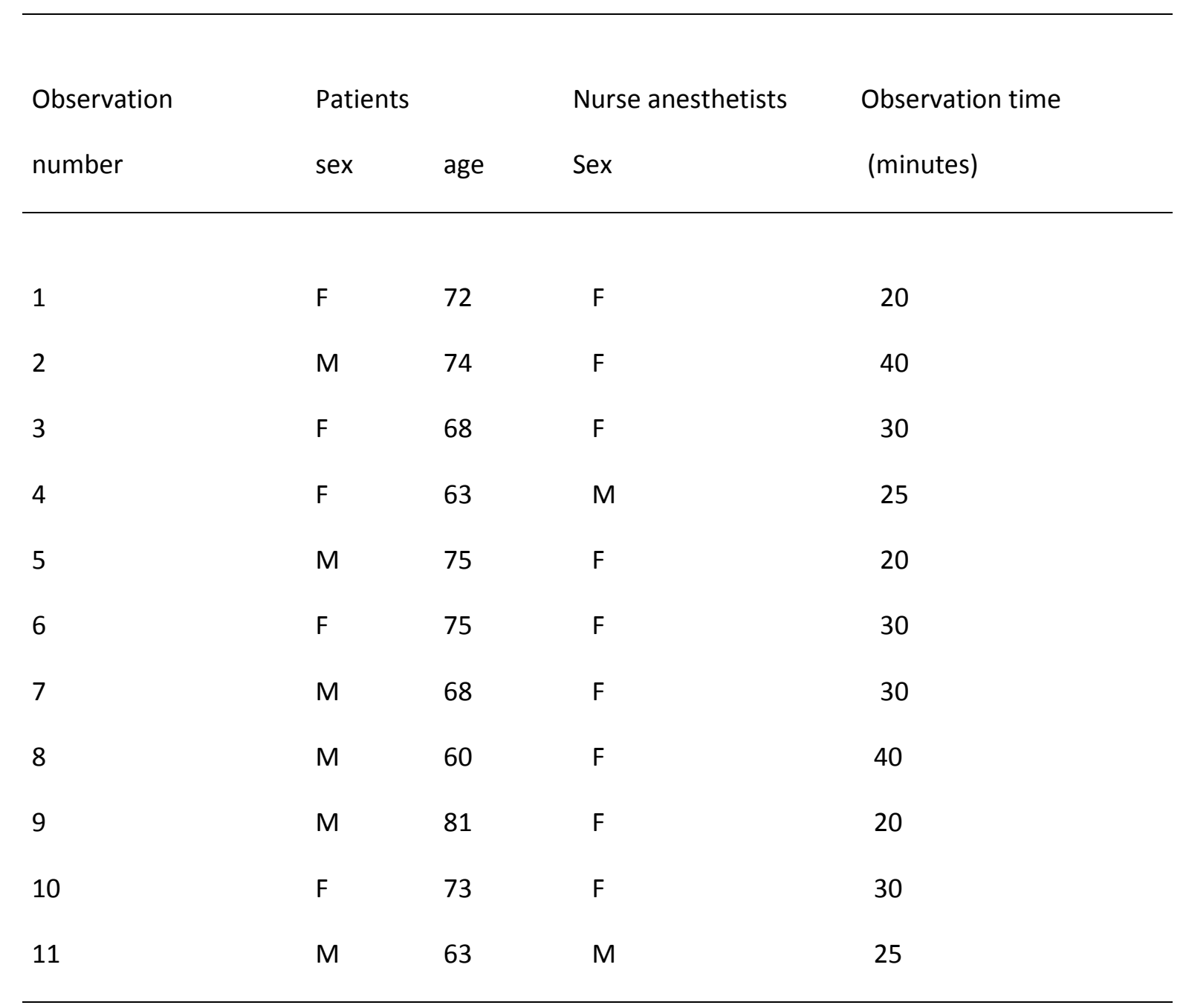

$\mathrm{F}=$ Female, $\mathrm{M}=$ Male

\section{Data analysis}

Data were analyzed using constant comparative method which is fundamental for grounded theory. The analyses were performed line-by-line through open coding and memos of the authors' reflections. Ideas were noted in order to increase theoretical sensitivity and establish a link between the coding and the emergence of a core category $[16,19]$. Through constant open coding of the data, substantive codes were generated and compared, aiming to find similarities and differences in the interactions. Related substantive codes were clustered and 
conceptualized into categories. The categories were related to each other and constantly refilled. Data were reviewed several times to fit and work in order to verify relevance and ensure that the data were empirically grounded [17]. A core category emerged from the content in the categories. In accordance with constant comparative method there was a continuous interplay between data analysis and data collection. Two of the authors, (RK, CU), analyzed the data together, while the other two authors (KB, GHF) analyzed the data independently of each other. Thereafter, all the authors agreed on the main codes and categories in order to explain the data and reach a higher level of abstraction [20]. After eight observations, three underlying categories and a core category emerged. Category headings were then discussed. Three further observations were made to assure saturation but no further codes or categories emerged. The process was constantly discussed by all authors to achieve credibility.

\section{FINDINGS}

\section{Reassurance - the core category}

The analysis of the observations showed that the meaning of the interaction between the patient and nurse anesthetist was reassurance, which emerged as the core category. Reassurance permeated the nurse-patient interaction throughout the preoperative preparation immediately prior to elective CABG surgery. Reassurance emerged from and was related to the three categories continuity, confirmation and control, which described the meaning of the interaction between the patient and nurse anesthetist (Fig 1). The core category reassurance was related to the category continuity through patients' feelings of security, resulting in a patient who was relaxed when meeting a nurse he/she had met before. Continuity was related to the category confirmation and influenced by the confirming atmosphere in the operating theatre, resulting in a sense of reassurance. Confirmation was in turn related to control as both 
the patient and nurse anesthetist relied on control during the whole preoperative preparation process. There was a relationship between control and reassurance through information that facilitated the understanding of the procedures.

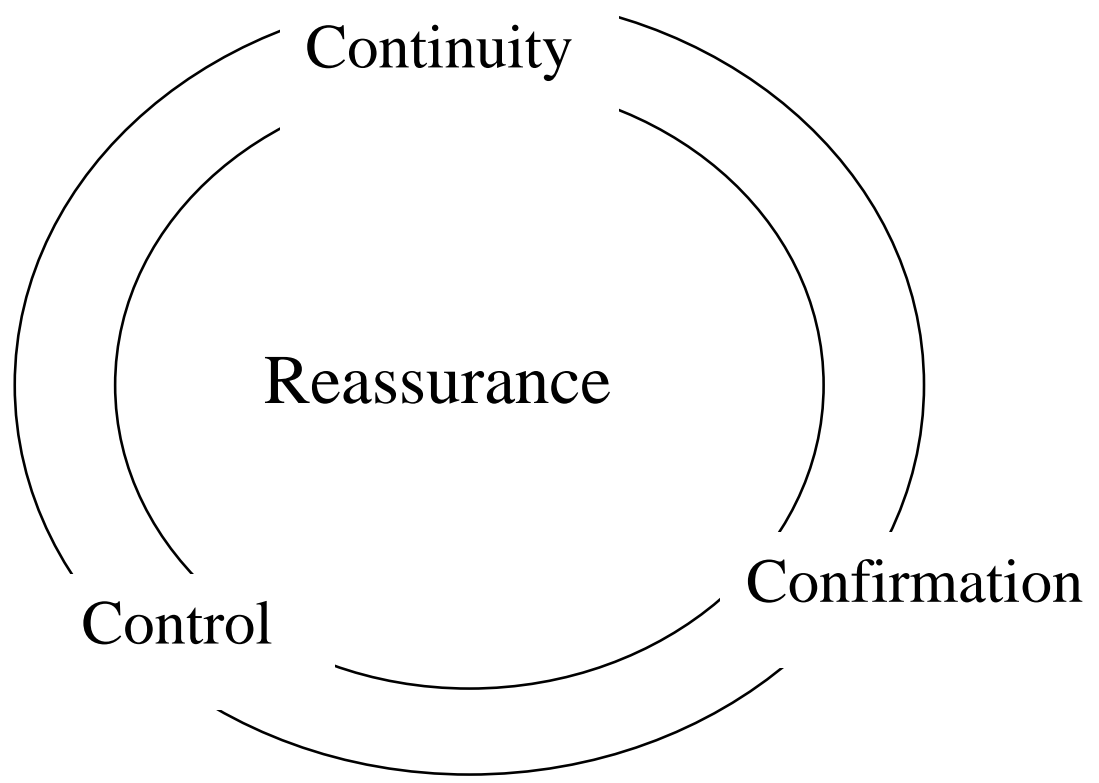

Figure 1.The core category, reassurance, with its underlying categories continuity, confirmation and control

Reassurance was established by the close and trustful interaction between the patient and nurse anesthetist, as well as the continuous focus on the patient. 


\section{Continuity}

In the operating theatre, a scene of well-planned and structured anesthesia nursing was derived from an effective continuity of care with a high level of safety. High quality anesthesia nursing emanated from safety which was expressed as a key feature of the professional nurse anesthetist. Effectiveness and continuity was characterized by a multi-task atmosphere where the nurse anesthetist was the coordinator.

The first interaction was the starting point for continuity and the patient-nurse interaction was purposive during the procedures prior to anesthesia induction. The time available for the nurse anesthetist to interact with the patient from entering the operation theatre until induction of anesthesia was limited, less than 40 minutes. Time restrictions characterized the interaction in which high quality nursing care was continuously conducted.

Moreover, continuity consisted of progression, manifested by the patient-nurse interaction. The patient needed to be assured that there would be continuous nursing care during the surgical procedure and that he/she would be safe.

Nurse anesthetist: 'I'm going to help you'. 'We are going to take care of you during your operation'. 'I'll come and see you after you have awakened in the intensive care unit'.

Patient: 'I trust that you'll safeguard me through the operation'.

The nurse confirmed the responsibility to stay by the patient throughout the operation and continuously keep contact to secure reassurance during the perioperative process. Hence, the patient was continuously guided and confirmed during the interaction. 


\section{Confirmation}

Reassuring interaction was characterized by mutual confirmation. The professional environment manifested itself as a confirming and trusting atmosphere. Prior to the patient entering the operation theatre, the nurse anesthetist had prepared medications and technical equipment in order to primarily focus on the arriving patient. Consequently, the patient met the waiting nurse anesthetist in a well prepared atmosphere which contributed to a sense of reassurance.

The communication was a confirmation process manifested both verbally and non-verbally. The synthesis of communication basically included a message and a response as an interplay between the nurse and the patient. The synthesis in the communicational strategy was launched when the patient entered the operating hallway, observed the surroundings and made eye contact with the nurse anesthetist.

Nurse anesthetist: 'Hi, welcome, my name is...... I'm a nurse anesthetist, and I am going to take care of you'.

The patient responded with a presentation of name, hands were shaken and eye contact was maintained. The patient responded:

'It feels good that you appear calm, because I'm not'. A silent moment with eye contact occurred. The nurse anesthetist responded, put her hand on the patient and asked: 'Did you get any sleep last night?' 
'Yes, as a matter of fact I did, I received medication which enabled me to have a good quality sleep'. 'That's great', responded the nurse anesthetist.

Prolonged information was seen as a sign of confirmation. The patient was continuously informed of what was going on during the perioperative period. The information video the patient had seen preoperatively was referred to and some information was repeated and explained. Some of the procedures during the operation that would be of importance postoperatively were explained in order to give the patient confirmation of the preoperative information. This confirmation meant safety for the patient who listened and sought for eye contact if not established and maintained. Even the postoperative period was trustfully confirmed.

Nurse anesthetist: 'I want you to know that you are going to receive a central venous catheter and a urine catheter after induction of anesthesia, which will safeguard you during your operation and the early postoperative stage'. The patient listened and maintained eye contact as the nurse anesthetist continued: 'After your surgery you will wake up in the intensive care unit, where intensive care nurses and physicians will monitor your health status and stay close to you'. The patient appeared relieved: 'Oh, that's good to know', while confirming smiles were exchanged.

The information transfer meant confirmation for both the patient and the nurse anesthetist. Satisfaction was expressed by the patient and there was a sense of grateful contact.

\section{Control}


For the nurse anesthetist, control consisted of the tasks performed when he/she checked, verified, regulated and modified responsibilities. Prepared equipment and medication were controlled in a safe and consistent atmosphere. The patients also needed a sense of control regarding information and understanding of the meaning of the procedures.

The patients experienced a new environment when they arrived in the operating unit. Accompanied by the ward nurse, they left their home ward where they had had some control and entered the new environment they had been informed about the previous day. The characteristics of the physical surroundings were manifested in an antiseptic, technical and surgical environment where the nurse anesthetist waited. Control started when the patient was introduced to this prepared environment and transferred into a supine position on an operating guerney. The nurse anesthetist carried out quality assurance by confirming the patient's identification, status and preoperative information while engaging in a relaxed conversation. The nurse anesthetist interrupted the ongoing conversation by informing the patient about the procedure in progress. The patient responded: 'Oh, I'll keep quiet so you can do your work'. The nurse: 'No, I didn't mean for you to be quiet, I just want you to know what I'm doing and why'. The patient: 'Ok'. The nurse anesthetist finished the described procedure and asked: 'What were you saying about your trip?' And the patient continued talking about a journey.

The patients were exposed to invasive procedures and were monitored. Furthermore, oxygen, fluids and antibiotics were administered. Induction of anesthesia, intubation and further preparations were controlled by the nurse.

Reassurance was established when the nurse anesthetist continuously confirmed the patient and thereby enabled the patient to feel in control. 


\section{DISCUSSION}

Our findings show that the core category was reassurance emerging from the categories continuity, confirmation and control, which contributes to the understanding of the meaning of the patient-nurse anesthetist interaction prior to elective CABG surgery. Reassurance has previously been described as a phenomenon that is an under-researched caring construct and relationally achieved in a satisfactory environment from interactional and communicational skills [21-24]. The Swedish description of competence for nurse anesthetists stresses the purpose of professional anesthesia nursing as being partly a contribution to reassurance during the short interaction time prior to surgery [1].

The underlying categories continuity, confirmation and control contributed to secure reassurance in the patient-nurse anesthetist interaction. In contrast, it has been shown that a technologically advanced caring environment may contribute to objectification of patients and nursing care, which may deprive patients of dignified subjectivity and separate nurses from their mission to care. A symbolic relationship between the technological environment and caring has previously been identified in acute settings [25, 26].

Seen as a model of holistic nursing the perioperative dialogue implements a 'continuous whole', achieved through patient-nurse anesthetist interaction [27, 28]. Continuity was experienced when the patient met a nurse he/she had met before. Being there for the patient throughout the operation ensured reassurance. These findings are supported by Soltner et al [29], who found that patients being cared for by just one emphatic anesthesiologist were more satisfied and rated quality of care higher. Emphatic behavior was also related to reduced levels of anxiety. Altogether, this demonstrates the importance of continuity in the interaction 
between the patient and anesthesia staff. Our findings also show another dimension of continuity, where continuity was manifested in an expected and well received patient, guided through the preoperative procedures by mutual confirmation.

Furthermore, the nurses' confirmative manner had a great influence on the patients' sense of safety, which is in line with nurses' experiences that creating a caring relationship is of importance to the perioperative dialogue. Through a caring relationship the nurse gets to know the patient as a person and simultaneously becomes a person to the patient. For the nurses, this developed caring competence [30]. This is in contrast to the nursing manner reported from an emergency department where the nurses had an instrumental behavior. Instrumental behavior lacks emotional involvement and may be seen as uncaring [5] and may contribute to existential anxiety among patients in a vulnerable situation. Thus, the anesthesia caring culture in this study seems to be positive as the patients expressed reassurance in the nursing care.

Experiencing safety and support has been described as essential for giving the patient an opportunity to achieve a sense of control during the preoperative phase [31]. The caring culture in this study was characterized by the nurse being prepared which gave her/him a chance to convey a sense of control to the patient. Patients' loss of control may contribute to perioperative stress, anxiety [32] and/or depression, which has been reported to be related to morbidity and mortality among patients undergoing CABG surgery [33-36]. Patients' psychosomatic complications and their association with morbidity and mortality challenge the nurse-patient interaction [33-36]. To reduce anxiety, the patients in this study needed control related to information and understanding of the perioperative course of events. 
Grounded theory was found to be an appropriate method for this observation study. Two authors (RK, CU) were present in the study environment during periods when no observations were made, in order to become familiar with the nurse anesthetists and the surroundings. According to GT [16], this strategy minimizes bias as the informants do not know when they are going to be observed.

At the time of data collection, two of the authors (RK, CU) had no knowledge of cardiothoracic nursing in an operating theatre or even of working as nurse anesthetists. The fact that the authors collecting data only had a minimal pre-comprehension of this patientnurse interaction is therefore seen as strengthening the trustworthiness of this study. In order to widen and achieve variation, a theoretical selection of patients and nurses was sought to secure trustworthiness of the study. Constant comparison of data secured the relevance, fit and workability of the categories and thus credibility was achieved. Trustworthiness was also ensured by all the authors analyzing the data and reaching consensus about the categories and the core category [16].

As the observations were unstructured, the idea was to catch everything that was going on during the observation [18]. In this study area there were also other professionals, such as assistant nurses, theatre nurses, surgeons and anesthesiologists, who sometimes interacted with the patient during the observations. These interactions were not the focus of the study and were disregarded in the observations, which could be seen as a limitation of the study [17]. Another limitation is the lack of interviews confirming the observations and further widening the categories. The lack of verbatim citations might also be seen as a study limitation. However, informants' statements contribute to the trustworthiness of the study. 
More men than women undergo elective $\mathrm{CABG}$, which is why there were fewer women than men included in this study. The rather high mean age is due to the fact that patients undergoing $\mathrm{CABG}$ are usually of an older age [37]. There were more women than men among the nurse anesthetists in the cardiothoracic operation unit, 15 women and 2 men, which explains why the study participant group was dominated by women.

\section{CONCLUSION}

Creating reassurance during the short interaction between the nurse anesthetist and the patient immediately prior to $\mathrm{CABG}$ surgery poses a challenge for the nurse anesthetist. Reassurance can be achieved through a well-structured performance of anesthesia nursing at the thoracic operating theatre, keeping the patient in focus and continuously giving the patient information during the preoperative preparation despite a highly technological and stressful environment. 


\section{REFERENCES}

1. Description of Competence for registered Nurses with graduate Diploma in Specialist Nursing - Anesthesia Nursing [cited 2012 February, http://www.swenurse.se.]

2. Eriksson K. Caring science in a new key. Nurs Sci Quart. 2002; 15(1):61-65.

3. Halldorsdottir S. Caring and uncaring encounters in nursing and health careDeveloping a theory. Thesis, Linköping University, Sweden; 1996.

4. Travelbee J. Interpersonal aspects of nursing. Philadelphia, PA: Davis; 1977.

5. Wiman E. Encounters between patients and staff in the emergency department . Thesis, Linköping University, Sweden; 2005.

6. Holmberg M, Fagerberg I. The encounter with the unknown: Nurses lived experiences of their responsibility for the care of the patient in the Swedish ambulance service. Int J Qual Stud Health Well-being. 2010; 5:5098 - DOI: 5010.3402/qhw.v5095i5092.5098.

7. Rudolfsson G, von Post I, Eriksson K. The expression of caring within the perioperative dialogue: A hermeneutic study. Int J Nurs Stud. 2007a; 44:905-915.

8. Schreiber R, MacDonald M. Keeping vigil over the patient: a grounded theory of nursing anaesthesia practice. JAN. 2010; 66(3):552-561.

9. Blumer H.Symbolic interactionism - perspective and method. Renewed 1998 edition. Los Angeles,CA: University of California Press; 1969.

10. Mead G. Mind, self \& society: from the standpoint of a social behaviorist. Chicago; The University of Chicago Press; 1934.

11. Wiman E, Wikblad K, Idvall E: Trauma patients' encounters with the team in the emergency department - A qualituative study. Int J Nurs Stud. 2007; 44:714-722.

12. Fredriksson L, Eriksson K. The ethics of the caring conversation. Nurs Ethics. 2003; 10(2):138-148.

13. Fleischer S, Berg A, Zimmerman M, Wüste K. Nurse-patient interaction and communication: A systematic literature review. J Publ Health. 2009; 17(5):339-353.

14. Teo K, Spoor M, Pressey T, Williamson H, et al: Impact of managed waiting for coronary artery bypass graft surgery on patients' perceived quality of life. Circulation. 1998; 98(19):1129-1134.

15. Råholm M, Lindholm L. Being in the world of the suffering patient: a challenge to nursing ethics. Nurs Ethics. 1999; 6(6):528-539.

16. Glaser B. Doing grounded theory: issues and discussions. Mill Valley, CA: Sociology Press; 1998.

17. Glaser B. Theoretical sensitivity. Mill Valley, CA: Sociology Press; 1978.

18. Lofland J, Snow D, Anderson L, Lofland L. Analyzing social settings - A guide to qualitative observation and analysis. 4 ed.. Wadsworth CA: Cengage learning; 2006.

19. Glaser B: Basics of grounded theory analysis: emergence vs forcing. Mill Valley, CA: Sociology Press; 1992.

20. Glaser B: The grounded theory perspective III: Theoretical coding. Mill Valley, CA: Sociology Press; 2005.

21. Fareed A. The experience of reassurance: patients' perspectivies. JAN. 1996; 23(2):272-279.

22. Johnson M, Goodacre S, Tod A, Read S. Patients' opinions of acute chest pain care: a qualitative evaluation of Chest Pain Units. JAN. 2009; 65(1):120-129.

23. Koeniger-Donohue R. Nurse practioner-client interaction as resource exchange: the nurse's view (NP-client interaction). J Clin Nurs. 2007; 16(6):1050-1060.

24. Pattison N, Dolan S, Townsend P, Townsend R. After critical care: a study to explore patients' experiences of a follow-up service. J Clin Nurs. 2007; 16(11):2122-2131. 
25. Almerud S, Alapack R, Fridlund B, Ekeberg M. Caught in an artificial split: A phenomenological study of being a caregiver in the technologically intense environment. Int Crit Care Nurs. 2008; 24(2):130-136.

26. Barnard A, Sandelowski M. Technology and human nursing care: (ir)reconciable or invented difference? JAN. 2001; 34(3):367-375.

27. Lindwall L, von Post I. Continuity created by nurses in the perioperative dialogue - a literature review. Scand J Care Sci. 2009; 23(2):395-401.

28. Rudolfsson G, von Post I, Eriksson K. The perioperative dialogue - holistic nursing in practice. Holist Nurs Pract. 2007b; 21(6):292-298.

29. Soltner C, Giquello J, Monrigal-Martin C, Beydon L. Continuous care and empathic anaesthesiologist attitude in the preoperative period: impact on patient anxiety and satisfaction. Br J Anaesth. 2011; 5:680-686.

30. Rudolfsson G, Ringsberg K, von Post I. A source of strength - nurses' perspectives of the perioperative dialogue. J Nurs Manage. 2003; 11:250-257.

31. Hankela S, Kiikkala I. Intraoperative nursing care as experienced by surgical patients. AORN J . 1996; 63(2):435-442.

32. Susleck D, Willocks A, Secrest J, et al; The perianesthesia experience from the patient's perspective. J PeriAnesthNurs. 2007; 22(1):10-20.

33. Blumenthal J, Lett H, Babyak M, et al: Depression as a risk factor for mortality after coronary artery bypass surgery. Lancet. 2003; 362:604-609.

34. Connerney I, Shapiro P, McLaughlin J, Bagiella E, Sloan R. Relation between depression after coronary artery bypass surgery and 12-month outcome: a prospective study. Lancet. 2001; 358:1766-1771.

35. Oxald M, Stubberfield J, Stuklis R, Edwards J, Wade T. Psychological risk factors for cardiac-related hospital readmission within 6 months of coronary artery bypass graft surgery. J Psychosom Res. 2006; 61(6):775-781.

36. Tully P, Baker R, Knight J. Anxiety and depression as factors for mortality after coronary artery bypass surgery. J Psychosom Res. 2008; 64(3):285-290.

37. Society of Thoracic Surgeons [cited 2012 February, www.sts.org.] 